\section{Obserwastăo das}

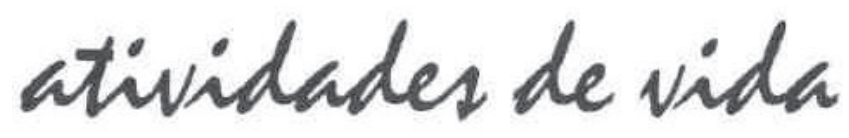

\section{diária através devideo.}

\author{
Anneliese Schonhorst Rocha* \\ Jorge Luiz de Souza**
}

\section{Abstract \\ Observation of daily chores through video}

The body postures intending to execute daily and labor chores such as sitting, grabbing, holding and moving objects, among others, are often responsible by the strain upon certain articulations and muscles, especially when done in a wrong way, not respecting the biomechanical principies. ${ }^{2}$ In order to efficiently check the body posture adopted under various everyday situations, an instrument of evaluation through video was designed and valided, based on the original test of dynamic posture, by Basler, Beisenherz-Hahn, Frank, Griss, Herda, Keller (1 993) adapted by Souza (1995), which allows us to judge the different movements and body postures under previously settled criteria. To obtain the validation of the instrument ten subjects were filmed, being later analyzed by three professional in dynamic body posture. The results of the statistical treatment showed a high index (Cronbach Alpha) of inner consistence for ali the analyzed criteria and a high correlation (Spearman) among the raters making this a reliable instrument.

\section{Resumo}

As posturas corporais assumidas para a execução das atividades de vida diária e nas tarefas laborais como sentar, pegar, carregar e trocar objetos de lugar, entre outras, muitas vezes são responsáveis pelos excessos provocados sobre determinadas articulações e grupos musculares, especialmente quando executadas de forma inadequada desrespeitando os princípios biomecânicos. ${ }^{1}$ Para verificar de forma efetiva a postura corporal adotada em diferentes situações do cotidiano, elaborou-se e validou-se um instrumento de avaliação através de vídeo, baseado no teste original de postura dinâmica, criado Basler, Beisenherz-Hahn, Frank, Griss, Herda, Keller, (1993) e adaptado por Souza (1 995), o qual permite o julgamento de diferentes movimentos e posições corporais, através de critérios previamente estabelecidos. Para se obter a validação do instrumento foi realizada uma filmagem de dez indivíduos, que foi analisada por dois profissionais da área de postura corporal. Os resultados do tratamento estatístico demonstraram alto índice (Cronbach Alpha) para consistência interna para todos os critérios analisados e uma alta correlação (Spearman) entre os avaliadores, tornando o instrumento fidedigno.

\section{INTRODUÇÃO}

A postura corporal tem sido estudada tanto no aspecto estático como no dinâmico, por ser considerada um fator determinante na eficiência mecânica dos movimentos ou no combate de problemas do sistema locomotor. O estudo da postura corporal é assunto bastante antigo. Referenciais a esse respeito podem ser encontrados em escritos bíblicos e na antigüidade. Hipócrates, por exemplo, denominou pela primeira vez o termo escoliose como um desvio lateral da coluna vertebral (Duque \& Brenzikofer, 1996). A preocupação, no momento, está voltada à postura corporal adotada durante às Atividades de Vida Diárias (AVD's), em que as pessoas executam as suas tarefas domésticas ou realizam as diferentes posturas do trabalho, sem preocuparem-se com a preservação das estruturas que compõem a coluna vertebral.

Nos últimos 15 anos, foram realizadas investiga- 
ções sobre a efetividade de programas especiais ${ }^{3}$ que envolvem o aprendizado e treinamento da postura corporal adotada pelas pessoas no dia-a-dia (Moffet, Chase, Portek \& Ennis, 1986; Otto, 1991; Kyburz \& Schwarz, 1992; Kaufmann, 1993). Concorda-se com Basler, BeisenherzHahn, Frank, Griss, Herda, Keller (1993) quando afirmam que os resultados apresentados pela maioria desses estudos precisam ser repensados, uma vez que os instrumentos de medida utilizados para avaliação da postura corporal não foram apresentados de uma maneira sistematizada e padronizada. Afirmam, estes autores, que uma forma efetiva para a avaliação da postura corporal dinâmica seria efetuá-la através de uma forma mais quantitativa. Por exemplo, a observação sistemática da postura utilizada para a realização dos movimentos diários, associada a um sistema de pontuação menos subjetivo, seria uma estratégia adequada.

Basler, Beisenherz-Hahn, Frank, Griss, Herda, Keller (1993) propuseram uma observação sistemática através da filmagem em vídeo, onde os critérios de avaliação foram previamente fixados. Embora tenha sido selecionada uma seqüência de movimentos diários e estabelecidos critérios para a sua avaliação, as quais foram julgadas por pessoas previamente treinadas, a escala adotada de 0-4 pode ser considerada pouco precisa para julgar cada movimento. Buscando uma menor subjetividade para a avaliação através da observação, Souza(1995) ampliou o método utilizado por Basler, Beisenherz-Hahn, Frank, Griss, Herda, Keller (1993), detalhando os critérios para a avaliação de cada gesto realizado. Neste estudo adaptou-se os critérios de Souza (1995) a uma seqüência de movimentos utilizados nas atividades diárias e laborais e padronizou-se uma forma de observação sistemática da postura corporal dinâmica. Diante destes aspectos, o presente artigo se propõe a apresentar a configuração, a metodologia e os coeficientes de validade e fidedignidade do instrumento.

\section{MATERIAIS E MÉTODOS}

$\mathrm{O}$ instrumento para avaliar as atividades de vida diária incluiu a avaliação de seis movimentos cotidianos, realizados sucessivamente numa rotina preestabelecida.

Os participantes analisados cumpriram uma rotina de ações, que foi filmada por um observador em uma sala previamente preparada para sua realização.
A amostra foi constituída de adultos de ambos os sexos, classificados como jovens e médios (Mosquera, 1987), com idade que variou de 20 à 50 anos. Esta amostra representava uma população de trabalhadores industriais, ligados às linhas de produção e às funções administrativas, os quais assumiam posições de trabalho avaliadas pelo instrumento.

Para a filmagem foram utilizados os seguintes equipamentos:

> Uma câmera de vídeo marca JVC, filmagem em VHS, modelo CGR AX 627, BN-V12U-NI-CD, com o recurso hiperzoom 14x para aproximação da imagem, possuindo dispositivo Auto-Light, acompanhada de carregador, eliminador, bateria e controle remoto.

$>$ Um tripé de apoio marca VIVITAR.

$>$ Uma mesa retangular com $80 \mathrm{~cm}$ de altura, aberta embaixo, para que pudessem ser filmados os membros inferiores dos participantes analisados.

$>$ Uma cadeira com $50 \mathrm{~cm}$ de altura, com um espaço de, aproximadamente, $20 \mathrm{~cm}$ de altura entre o encosto e o assento.

$>$ Um objeto com base retangular com $20 \mathrm{~cm}$ de largura e $15 \mathrm{~cm}$ de altura e pesando $20 \mathrm{Kg}$.

$>$ Um banco pequeno, sem encosto, com $50 \mathrm{~cm}$ de altura.

Colocada sobre o tripé de apoio, a filmadora pôde ser movida durante a filmagem, de um lado para o outro, para acompanhar os movimentos do participante em avaliação. O observador instalou a filmadora no local em que os ângulos recomendados pudessem ser visualizados.

Foram analisadas seis posições, nos seguintes ângulos:

$>$ Ato de sentar: vista lateral.

$>$ Posição sentada no banco: vista lateral.

> Posição sentada na cadeira: vista lateral.

$>$ Pegar um objeto do solo: vista lateral.

$>$ Carregar um objeto ${ }^{4}$ : vista lateral e anterior.

$>$ Trocar o objeto de lugar: vista lateral.

A rotina adotada foi a seguinte, e está ilustrada esquematicamente no diagrama da Figura 1: 
1. O participante posicionou-se numa distância de 6 $\mathrm{m}$ à frente da filmadora, na posição ortostática;

2. Após o sinal do observador, o participante virou-se de frente para o objeto, previamente colocado ao seu lado, e o apanhou.

3. Virou-se novamente para a filmadora e deslocouse em sua direção, carregando o objeto até o ponto designado "XI" marcado no solo;

4. Quando chegou neste ponto virou-se de lado para a filmadora ( $1 / 4$ de giro à esquerda) e continuou carregando o objeto até o ponto designado "X2" colocado no solo à sua frente, ao lado da mesa.

5. Virou-se de lado para a filmadora (1/4 de giro à esquerda), largou o objeto no solo e caminhou em direção à cadeira colocada na ponta da mesa.

6. Sentou-se na cadeira e permaneceu sentado de lado para a filmadora, durante 30 segundos (o observador que estava filmando usou, neste momento, o recurso do zoom da filmadora, direcionando para a pelve do participante);

7. Após permanecer sentado, o participante levantou- se e caminhou em direção ao ponto "X2". Posicionou-se de frente para o objeto e de lado para a filmadora. Apanhou o objeto e o colocou sobre a mesa;

8. O participante deixou o objeto em cima da mesa, caminhou até o banquinho colocado à frente da filmadora, fez um meio giro e permaneceu em pé, durante 10 segundos, de costas para o banquinho e de lado para a filmadora. Sentou e permaneceu nesta posição, aproximadamente, 30 segundos.

9. O participante levantou, deslocou-se novamente até a mesa e apanhou o objeto que havia colocado, anteriormente, sobre ela.

1O.Na última etapa, caminhou até o lugar de origem e recolocou o objeto no mesmo local onde iniciou a rotina.

A rotina foi explicada antes de iniciar a filmagem. Durante a sua realização o observador forneceu para os participantes as instruções necessárias sobre a seqüência de movimentos.

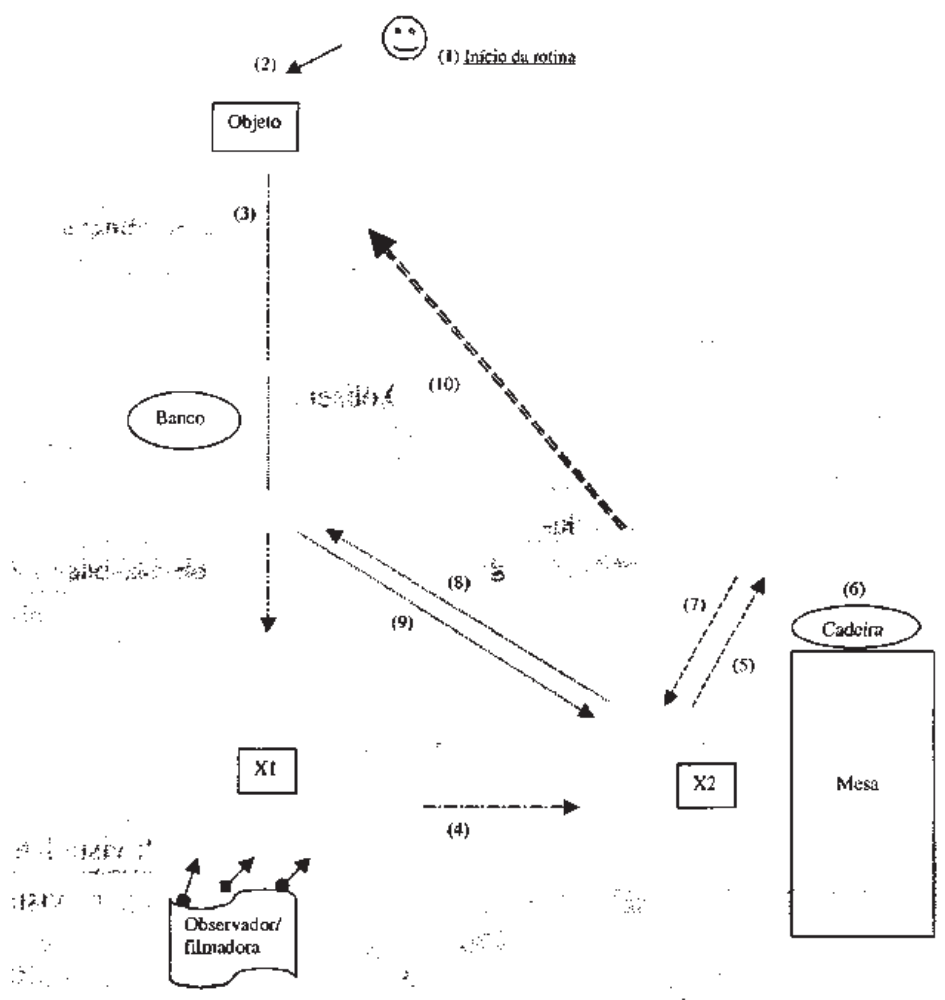

Figura 1. Execução da rotina estabelecida para o instrumento de avaliação da postura corporal dinâmica. 
Após a filmagem concluída, a postura corporal dinâmica foi analisada conforme critérios pré-estabelecidos, descritos na Tabela 1

\section{VALIDADE DE CONTEÚDO, CONSISTÊNCIA INTERNA E} FIDEDIGNIDADE DO INSTRUMENTO

A rotina como está descrita foi modificada da ori- ginal, criada por Basler, Beisenherz-Hahn, Frank, Griss, Herda e Keller (1993) e adaptada por Souza (1995) com coeficiente de objetividade $\mathrm{r}=0,91$, tendo reduzido o número de posições analisadas e estruturando os itens de forma mais específica.

Para a validação de conteúdo, o instrumento foi apresentado a três profissionais da área postural, os quais afirmaram ser este, coerente para análise da postura dinâ-

Tabela 1. Posições analisadas e critérios para o instrumento de avaliação da postura corporal dinâmica.

\begin{tabular}{|c|c|c|}
\hline POSIÇÃO ANALISADA & NOTAS & CRITÉRIOS PARA ANÁLISE \\
\hline $\begin{array}{l}\text { 1. Postura ao pegar um objeto do } \\
\therefore\end{array}$ & $\begin{array}{l}1 \\
1 \\
1\end{array}$ & $\begin{array}{l}\text { Manutenção das curvaturas da coluna (dorsal e lombar); } \\
\text { Membros inferiores afastados (igual ou além da linha do } \\
\text { quadril); } \\
\text { Objeto próximo ao corpo; } \\
\text { Flexão dos joelhos (aproximadamente } 90^{\circ} \mathrm{com} \text { membros } \\
\text { inferiores simétricos). }\end{array}$ \\
\hline 2. Postura ao carregar um objeto & $\begin{array}{l}1 \\
1 \\
1 \\
1\end{array}$ & $\begin{array}{l}\text { Não elevar os ombros (vista anterior); } \\
\text { Manutenção das curvaturas da coluna (dorsal)- (vista } \\
\text { lateral); } \\
\text { Objeto próximo ao corpo (vista lateral); } \\
\text { Manutenção da pelve na posição neutra. }\end{array}$ \\
\hline $\begin{array}{c}\text { 3. Postura ao trocar um objeto de } \\
\text { lugar } \\
\text { and }\end{array}$ & $\begin{array}{l}1 \\
1 \\
1\end{array}$ & $\begin{array}{l}\text { Torção correta de tronco (virar de frente ou girar tronco e } \\
\text { quadril juntos); } \\
\text { Manutenção das curvaturas da coluna (dorsal); } \\
\text { Objeto próximo ao corpo; } \\
\text { Membros inferiores afastados. }\end{array}$ \\
\hline $\begin{array}{l}\text { 4. Postura durante a posição } \\
\text { sentada (cadeira) }\end{array}$ & $\begin{array}{l}1 \\
1 \\
1\end{array}$ & $\begin{array}{l}\text { Manutenção das curvaturas da coluna(dorsal); } \\
\text { Próximo à mesa; } \\
\text { Posicionamento neutro da pelve (apoio nos ísquios); } \\
\text { Membros inferiores afastados (igual ou além a linha do } \\
\text { quadril). }\end{array}$ \\
\hline 5. Ato de sentar & $\begin{array}{l}1 \\
1 \\
1 \\
1\end{array}$ & $\begin{array}{l}\text { Manutenção das curvaturas da coluna (cervical e dorsal); } \\
\text { Báscula anterior com flexão de quadril; } \\
\text { Inclinação do tronco à frente; } \\
\text { Membros inferiores afastados (igual ou além a linha do } \\
\text { quadril). }\end{array}$ \\
\hline $\begin{array}{l}\text { 6. Posição durante a posiçăo } \\
\text { sentada (banco) }\end{array}$ & $\begin{array}{r}1 \\
1 \\
1 \\
1 \\
1 \\
1\end{array}$ & $\begin{array}{l}\text { Manutenção das curvaturas da coluna (dorsal); } \\
\text { Posicionamento neutro da pelve (apoio nos ísquios); } \\
\text { Membros inferiores afastados (igual ou além a linha do } \\
\text { quadril); } \\
\text { Sola dos pé apoiados em uma base ou no solo. }\end{array}$ \\
\hline
\end{tabular}


mica, já que os critérios de avaliação são baseados nos princípios biomecânicos referidos pela literatura.

Para verificação da consistência interna e da fidedignidade, o instrumento foi aplicado à amostra descrita anteriormente. Foram filmados 10 indivíduos da amostra referida executando a rotina de ações já adaptada.

A filmagem foi analisada por dois profissionais da área postural, cada um atribuindo notas de 0 a 4 para cada posição analisada.

Para a verificação da consistência interna e da coerência entre o teste e o reteste, cada profissional analisou a filmagem 2 vezes (teste-reteste), com um intervalo de 10 dias entre uma análise e outra. Verificou-se o "coeficiente alfa" (Cronbach, 1969) entre as notas atribuídas para cada posição analisada pelo instrumento (ver Tabela 2).

Os dados apontaram um coeficiente alfa elevado ${ }^{6} \mathrm{e}$ aceitável estatisticamente no teste e no re avaliadores.

Os valores da correlação intra-classe o reteste, na soma das notas de cada avalic mostrados na Tabela 3 (ver).

Os resultados mostram uma correli estatisticamente, entre o teste e o reteste dos do

Para investigar a fidedignidade do ir ram realizadas 2 filmagens do mesmo suj intervalo de 10 dias entre uma e outra. $\mathrm{O}$ ar do acima analisou a filmagem e atribuiu $\mathrm{r}$ para cada posição analisada pelo instrum uma correlação bivariada de Spearman, co notas, entre o teste (filmagem 1) e o reteste em que se obtiveram os resultados apontado

Os valores acima demonstram que $\mathrm{h}$

Tabela 2. Resultados do teste e reteste das notas de todas as posições pertencentes ao teste de postura dinâ

\begin{tabular}{|c|c|c|c|c|c|c|c|}
\hline \multicolumn{4}{|c|}{ Avaliador 1} & \multicolumn{4}{|c|}{ Avaliador 2} \\
\hline Teste & Reteste & $n$ & $\begin{array}{l}\text { N. }{ }^{\circ} \text { de } \\
\text { itens }\end{array}$ & Teste & Resteste & $\mathrm{N}$ & $\begin{array}{l}\mathrm{N}^{\circ}{ }^{\circ} \mathrm{de} \\
\text { itens }\end{array}$ \\
\hline$\alpha=0.7841$ & $\alpha=0,8027$ & & & $\alpha=0,8266$ & $\alpha=0.7943$ & & \\
\hline$p=0,0023^{*}$ & $\mathrm{p}=0,0000^{*}$ & 10 & 24 & $\mathrm{p}=0,0009^{*}$. & $\mathrm{p}=0,0004 *$ & 10 & 24 \\
\hline
\end{tabular}

Coeficiente alfa $(\alpha)$, nível de significância (p) e número de participantes (n).

${ }^{*} \mathrm{p}<0.05=$ significativo. $\mathrm{p}>0.05=$ não significativo

Tabela 3. Resultados do teste e reteste da soma total das notas do teste de postura dinâmica.

\begin{tabular}{|c|c|c|c|c|c|}
\hline \multicolumn{3}{|c|}{ Avaliador 1} & \multicolumn{3}{|c|}{ Avaliador 2} \\
\hline Correlação Intra-Classe & $\mathrm{n}$ & $\mathrm{N} .^{\circ}$ de itens & Correlação Intra-Classe & $\mathrm{n}$ & N. ${ }^{\circ}$ de ite \\
\hline$r=0,9936$ & & & $\mathrm{r}=0,816 \mathrm{I}$ & & \\
\hline $\mathrm{p}=0,0000^{\text {疋 }}$ & 10 & 2 & $\mathrm{p}=0,0070^{*}$ & 10 & 2 \\
\hline
\end{tabular}

Coeficiente da correlação intraclasse ( $\mathrm{r}$ ), nível de significância (p) e número de participantes (n).

* $\mathrm{p}<0.05=$ significativo, $\mathrm{p}>0.05=$ não-significativo.

Tabela 4. Resultado da correlação de spearman e seu nível de significância.

\begin{tabular}{|c|c|c|c|}
\hline \multirow{2}{*}{$\cdots$} & \multicolumn{3}{|c|}{ Teste a Reteste } \\
\hline & $n=10$ & $r=0,988$ & $\mathrm{p}=0,000^{*}$ \\
\hline
\end{tabular}


cia entre as notas atribuídas pelo avaliador nas duas situações de filmagem.

O software utilizado para tratamento estatístico dos dados foi o SPSS versão 7.0.

\section{DiSCUSSÃO DOS RESULTADOS}

A consistência interna do instrumento, através do Cronbach Alpha, aponta que o contruto, neste caso a postura dinâmica, pode ser analisado objetivamente, baseado na coerência dos critérios pré-estabelecidos, de forma quantitativa. Na testagem da correlação entre as notas dos dois profissionais, obteve-se uma coerência entre as análises $(\mathrm{r}=0,9936$ e $\mathrm{r}=0,8161)$, apesar de não serem próximas. Esses valores demonstram o quanto é importante que este instrumento seja aplicado por especialistas em postura dinâmica ou por observadores preparados para este fim, conhecedores dos critérios que o compõe, para que seja feita uma análise correta. Basler, Beisenherz-Hahn, Frank, Griss, Herda, Keller (1993) detectaram que observadores especialistas em avaliação postural apresentam maior empenho que os não especialistas.

A grande vantagem deste instrumento é a facilidade de sua operacionalização, já que, é possível filmar o indivíduo fora do laboratório, estando ele no seu próprio ambiente, em casa ou no trabalho. Inclusive é interessante verificar se o indivíduo mantém as posturas adotadas durante a rotina de ações no seu dia-a-dia, com o intuito de averiguar se tais posturas são naturais ou se ele as modificou somente durante a execução da rotina.

É necessário destacar que neste instrumento são analisados alguns dos movimentos cotidianos, como sentar, pegar um objeto do solo etc, outros, porém, como caminhar, ficar parado em pé, deitar etc, não o compõe, por enquanto. As posições que constituem o instrumento atual foram escolhidas entre outras por serem usadas repetidamente, tanto no trabalho como em casa.

Com estes resultados, o instrumento para análise das atividades de vida diária através de vídeo, demostra ser coerente e fidedigno, para estudos relacionados à postura corporal dinâmica.

Para trabalhos posteriores sugere-se a ampliação do presente instrumento, a fim de reunir outros movimentos c posições usados no dia-a-dia. Esta mesma avaliação também poderá ser feita por novos instrumentos como o software peak-performance através de avaliação computadorizada.

\section{REFERÊNCIAS BIBLIOGRÁFICAS}

BASLER, H.; BEISENHERZ-HAHN, A.; FRANK, R; GRISS, C; HERDA, C; KELLER, S. Rücken starken- Konzept und Evaluation einer Rückenschule für den Arbeitsplatz. Der Schmerz, 7, 268-279, 1993.

CRONBACH, L. J. Essentials of phychological testing. 3.ed. Nova York: Haper \& Row, 1969.

DUQUE, C. \& BRENZIKOFER, R. Forma geométrica da Coluna Vertebral - Obtenção de um banco de dados. In: Anais do VII Congresso Brasileiro de Biomecânica. Campinas, 1996.

FRACCAROLI, J. L. Biomecânica: uma análise dos movimentos. 2.ed. Rio de Janeiro: Cultura Médica, 1981.

KAPANDJ, I. A. Fisiologia articular, v. 3. São Paulo: Manole, 1990.

KAUFMANN, A. Verhaltenseifekte einerpräventiven Rückenschule - eine Evaluationsstudie. Unverôffentl. Diplomarbeit im ASG, ISSW Heidelberg, 1993.

KENDALL, F.R, McCREARY, E.K., PROVANCE, RG. Provas e funções musculares, 4.ed. São Paulo: Manole, 1995.

KYBURZ, G. \& SCHWARZ, J. Das Rückenprogramm der AOK Pforzheim - eine Evaluationsstudie im Rahmen der betrieblichen Prävention. Bericht zum 6. Heidelberger Fachgesprüch — Effektivitãt von Rückenschule. ISSW Heidelberg, 1992.

MOFFET, J. A. K., CHASE, S. M., PORTEK, I. \& ENNIS, J. R. A Controlled, Prospective Study to Evaluate the Effectivness of a Back School in the Relief of Chronic Low BackPain. Spine, 11, 120-122, 1986.

MOSQUERA, J. Vida adulta. 3.ed. Porto Alegre: Sulina, 1987.

NORDIN, M., FRANKEL, V.H. Basic biomechanics of the músculoskeletal system. 2.ed. Nova York/EUA: Lea \& Febiger, 1989.

OTTO, D. Das Azubi-Programm hei Siemens - eine Evaluationsstudie. Bericht zum 2. Karlsruher Symposium zur Prãventiven Gesundheitsfõrderung — "Gesunder Rücken am Arbeitsplatz". ISSW Karlsruhe, 1991.

SOUCHARD, E. Reeducação postural global. São Paulo: Martins Fontes, 1984

SOUZA, J. L. Untersuchungen zur Wirksamkeit von Bewegungsprogrammen bei Rückenbeschwerden. Inauguraldissertation zur Erlangung des Doktorgrades an der Fakultãt für Sozial- und Verhaltenswissenschaften der Ruprecht-KarlsUniversitat Heidelberg, 1995. 


\section{NOTAS}

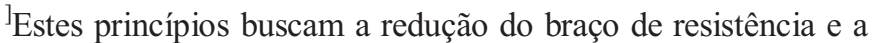
distribuição do esforço muscular em diferentes articulações, sendo baseados nas leis gerais da física e visam o movimento adequado. Este movimento adequado será aquele que, entre determinados limites, não prejudique o organismo, não criando sobrecargas funcionais ou condições em que, a curto ou a longo prazo, possam originar processos patológicos, buscando uma posição mais estável e de equilíbrio (Fraccaroli,1981; Souchard, 1984; Nordin e Frankel, 1989).

${ }^{2}$ These principies seek the reduction of the resistance arm and the distribution of the muscular effort in different articulations, being based on the general laws of physics and they aim at the correct movement. The correct movement is the one that within certain limits does not harm the organism, thus not creating functional overburdens or conditions that in the short or long run may originate pathological processes, fetching balance (Fraccaroli,1981; Souchard, 1984; Nordin e Frankel, 1989).

${ }^{3}$ Programas de treinamentos visando a melhora da postura corporal dinâmica em que os participantes recebem informações gerais para a execução adequada das Atividades Diárias (sentar, levantar e carregar objetos, rotações da coluna etc).

${ }^{4}$ Carregar o objeto por 3 metros.

${ }^{5}$ Pode-se caracterizar como báscula anterior, o avanço das espinhas ilíacas ântero-superiores em relação ao púbis (Kapandji, 1990 e Kendall, Mccreary, Provance 1995).

${ }^{\circ} \mathrm{O}$ coeficiente alfa considerado ideal é 1 e aceitável se maior queO,7(Levin,1987).

\section{UNITERMOS}

Postura dinâmica; atividades de vida diária; avaliação; filmagem.

*Anneliese Schonhorst Rocha ê Mestre em Ciências do Movimento Humano pela ESEF / UFRGS.

**Jorge Luiz de Souza é Doutor pela Universidade de

Heidelberg, Alemanha, e professor titular da ESEF/ UFRGS. 